\title{
Recoding preventive exposures to get valid measures of interaction on an additive scale
}

\author{
Mirjam J. Knol · Tyler J. VanderWeele
}

Received: 26 May 2011/Accepted: 17 August 2011/Published online: 4 September 2011

(C) The Author(s) 2011

Ding et al. [1] recently published a paper on GEIRA, a geneenvironment and gene-gene interaction research application. The authors developed a program in SAS and R to calculate interactions on an additive and multiplicative scale. We want to compliment the authors on providing such a useful tool, which may accommodate more frequent calculation of interaction on an additive scale. However, there is one flaw in the program, which the authors only partly acknowledge, that we would like to address. Measures of interaction on an additive scale, such as the proportion attributable to interaction (AP), cannot be validly calculated if any of the odds ratios of the independent and joint effects is smaller than 1, i.e. preventive [2]. The authors state in the discussion section that: "[] the OR associated with the genetic factor might still be less than 1 among subjects unexposed to the environmental factor. In this case, the interpretation for interaction should be cautious". We think that 'caution' is not enough; in this case, the calculations should just not be performed. Fortunately, there is an easy solution to get valid estimates for measures of interaction on an additive scale when having preventive exposures by appropriately recoding one or both of the exposures. We have described this in detail elsewhere for general measures of additive interaction [2]. Tests for specific forms of antagonism may, however, require different forms of recoding [3].

The reason why using odds ratios smaller than 1 gives wrong results in the measures of interaction on an additive scale is because an odds ratio is restricted between 0 and 1

M. J. Knol ( $\square)$

Julius Center for Health Sciences and Primary Care, University

Medical Center Utrecht, Utrecht, The Netherlands

e-mail: m.j.knol@umcutrecht.nl

T. J. VanderWeele

Departments of Epidemiology and Biostatistics, Harvard School

of Public Health, Boston, MA, USA for a preventive factor while it can go from 1 to infinity for a risk factor. For example, an odds ratio of 0.60 means a relative risk reduction of $40 \%$, whereas the inverse (1/ $0.60=1.67$ ) means a relative increase in risk of $67 \%$. If 0.60 is used in calculating a measure of additive interaction this leads to different results than if 1.67 is used in calculating the interaction. Moreover, with preventive exposures, measures such as the relative excess risk due to interaction or the attributable proportion can give results that are in the opposite direction of other additive interaction measures such as the synergy index [2].

A simple solution for the problem is recoding the variables in such a way that the stratum with the lowest risk when both factors are considered jointly becomes the reference category. The result of this recoding is that for one or both factors the absence of the exposure (rather than the presence) becomes the risk factor for the outcome. If factors are recoded one at a time, rather than jointly as we suggest, this can again result in invalid measures of interaction on an additive scale [2].

The authors of GEIRA might consider incorporating a recoding scheme in their program to prevent mistakes from being made when calculating measures of interaction on an additive scale with odds ratios smaller than 1. Alternatively, users of GEIRA could carry out this recoding themselves and apply GEIRA subsequently. Caution is always needed in interpreting the interaction after recoding as the exposure is changed to its opposite.

\section{References}

1. Ding B, Kallberg H, Klareskog L, Padyukov L, Alfredsson L. GEIRA: gene-environment and gene-gene 
interaction research application. Eur J Epidemiol 2011; 26: $557-561$.

2. Knol MJ, Vanderweele TJ, Groenwold RH, Klungel $\mathrm{OH}$, Rovers MM, Grobbee DE. Estimating measures of interaction on an additive scale for preventive exposures. Eur J Epidemiol 2011; 26: 433-438.

3. Vanderweele TJ, Knol MJ. Remarks on antagonism. Am J Epidemiol 2011; 173: 1140-1147.

\section{The Authors' Reply}

We are grateful to Knol and VanderWeele for having drawn attention to an ambiguity when calculating measures of interaction on an additive scale when any of the interacting factors is preventive [1]. If factors are coded one at a time without considering their joint effect, different measures of interaction may give incompatible results. We agree that our comments of this problem were incomplete and unsatisfactory in our article [2]. Knol and VanderWeele has timely shown that recoding of preventive factors by choosing the stratum with the lowest risk when both factors are considered jointly as the reference category solves the problem [3]. We agree that their suggestion solves the problem and will generate valid measures of interaction on an additive scale. We have now updated the GEIRA program to automate the recoding procedure
(GEIRA version 2.0). A flag variable "recoding" will indicate which factor (environment, gene or both) was recoded.

Open Access This article is distributed under the terms of the Creative Commons Attribution Noncommercial License which permits any noncommercial use, distribution, and reproduction in any medium, provided the original author(s) and source are credited.

\section{References}

1. Knol MJ, VanderWeele. Recoding preventive exposures to get valid measures of interaction on an additive scale. Eur J Epidemiol. 2011.

2. Ding B, Källberg H, Klareskog L, Padyukov L, Alfredsson L. GEIRA:gene-environment and genegene interaction research application. Eur J Epidemiol. 2011. Jul;26(7):557-61.

3. Knol MJ, Vanderweele TJ, Groenwold RH, Klungel $\mathrm{OH}$, Rovers MM, Grobbee DE. Estimating measures of interaction on an additive scale for preventive exposures. Eur J Epidemiol. 2011 Jun;26(6):433-8.

Bo Ding, Henrik Källberg, Lars Alfredsson Institute of Environmental Medicine, Karolinska Institutet, Box 210, 17177 Stockholm, Sweden 